\title{
Dynamic characteristics of multi-walled carbon nanotubes under a transverse magnetic field
}

\author{
S LI, H J XIE and X WANG* \\ School of Naval Architecture, Ocean and Civil Engineering, State Key Laboratory of Ocean Engineering, \\ Shanghai Jiaotong University, Shanghai 200030, P. R. China
}

MS received 5 July 2010; revised 28 November 2010

\begin{abstract}
This paper reports the results of an investigation into the effect of transverse magnetic fields on dynamic characteristics of multi-walled carbon nanotubes (MWNTs). Couple dynamic equations of MWNTs subjected to a transverse magnetic field are derived and solved by considering the Lorentz magnetic forces induced by a transverse magnetic field exerted on MWCNTs. Results show that the transverse magnetic field exerted on MWNTs makes the lowest frequency of the MWNTs nonlinearly decrease and the highest frequency, changeless. When the strength of applied transverse magnetic fields is larger than a given value the two walls of MWNTs appear in the radial and axial coaxial vibration phenomena.
\end{abstract}

Keywords. Dynamic characteristics; multi-walled carbon nanotubes; transverse magnetic field; van der Waals force.

\section{Introduction}

Since the discovery of carbon nanotubes (CNTs) (Iijima 1991), extensive research related to the carbon nanotubes in the fields of chemistry, physics, materials science and engineering, and electric engineering has been carried out $(\mathrm{Ru}$ 2000; Steven 2001; Yakobson and Avouris Phaedon 2001). Because of its superior electronic and mechanical properties over any known material, many stimulated extensive studies such as parts of nano-devices, nano-switch, nano-sensors and high frequency oscillators have been presented (Avouris et al 1999; Dresselhaus et al 2001; Tu and Yang 2002; Lau 2003; Han et al 2005).

From previous results (Heremans et al 1994; Langer et al 1996; Kandaa et al 2002; Macedo and Souza 2004; Reddy et al 2006; Qin et al 2009), it is seen that structural parameters of carbon nanotubes such as diameter, helicity and layer numbers of concentric cylinders, strongly change the electronic properties of carbon nanotubes, and the magnetic susceptibility of carbon nanotubes is strongly dependent on the presence of a strongly affecting magnetic field. The magnetic field dependence of electron transport in highly resistive metal-on-tube metal/multi-wall carbon nanotube/metal structures is described elsewhere (Kandaa et al 2002). Macedo and Souza (2004) described the magnetic thermodynamic properties of carbon nanotubes using a single Hubbard model at half-filling, and gave the temperature and field dependence of the magnetic susceptibility, subtracting the diamagnetic contribution of the considered nanotube

*Author for correspondence (xwang@ @stu.edu.cn) models in very good qualitative agreement with the experimental data for a mixture of nanotubes of different diameters. The results of an investigation into the magnetic properties of these metal-filled MWNTs by vibrating sample magnetometer is presented elsewhere (Reddy et al 2006). Qin et al (2009) fabricated magnetic nanocomposites through embedding modified carbon nanotubes into epoxy resins, and investigated magnetic properties by vibrating the sample magnetometer.

From the literature (Kibis 2002), it is seen that an electron energy spectrum in chiral carbon nanotubes under an external symmetrically magnetic field is asymmetrically relative to inversion of electron wave vector. Due to this asymmetry, electronic properties of the nanotubes are non-equivalent for mutually opposite directions. Thus, a change of magnetic field results in oscillations of nanotubes. Fedorov et al (2010) investigated the effect of magnetic field on the transfer characteristic of a device fabricated in a configuration of a field-effect transistor with a conduction channel formed by a semiconducting multiwalled carbon nanotube. It is unambiguously seen from their results that a magnetic field suppresses the bandgap of nanotube. Electromagnetic characteristics and microwave absorbing properties of MWNTs filled with $\mathrm{Er}_{2} \mathrm{O}_{3}$ were investigated in the frequency range 2-18 GHz (Zhang et al 2008). It is seen from their investigations that because the complex permittivity and electric loss tangent of $\mathrm{Er}_{2} \mathrm{O}_{3}$-filled MWNTs present a decreasing trend while the complex permeability and magnetic loss tangent are larger than those of the unfilled MWNTs, the modified MWNTs possess much broader absorbing bandwidth and larger reflectivity than those of unfilled MWNTs. 
One of the common characteristics of those nano-electron devices is that it often couples in magnetic field. So, understanding the effect of magnetic field on mechanical and physical properties of CNTs is essential and may give a useful help in applications for nano-electric engineering. However, the effect of magnetic field on vibration frequencies and modes of MWNTs have not been reported in literature so far.

It is seen in the literature that to analyse and interpret the mechanical performance of CNTs, experimental methods (Li and Bhushan 2002; Li et al 2004), molecular dynamics methods (MD) (Nardelli et al 1998; Wang and Ni 2003), and continuum mechanics model (Cai and Wang 2006; Wang and Cai 2006) are frequently used. However, it is difficult to investigate physical properties of CNTs directly using the experimental methods due to limited number of specimens available and difficulties in manipulating the CNTs having smaller tube diameter and length. MD simulation is an effective technique that simulates accurately the physical properties of structures at atomic scale, in which the computational problem is that the time steps involved in the MD simulations are limited by the vibration modes of the atoms to be of the order of femto-seconds $\left(10^{-15}\right)$. So even after a million time steps, we can reach only the nanosecond range, in which period most of the mechanical, physical, or magnetic events have not even started (Saito et al 1998). Using continuum mechanics methods, Wang et al (2004) reported results of an investigation into the RBMs of CNTs, in which the results obtained were in good agreement with molecular dynamic (MD) simulation results (Popov and Henrard 2002) with relative errors $<5 \%$.

In this paper, the multiple-shell model is further employed to study the effect of transverse magnetic field on vibration frequencies and modes of MWNTs for which detailed experimental data or MD simulations are not yet available in the literature. Results carried out show that the transverse magnetic field exerted on MWNTs makes the lowest frequency of the MWNTs non-linearly decrease and the highest frequency, changeless, two walls of MWNTs appear in larger relative radial motion and axial motion when the strength of transverse magnetic fields exterted on the MWNTs is larger than a given value, and the relative radial motion amplitude between two walls of the MWNTs is less than its relative axial motion amplitude.

New features for the vibration frequencies and modes of MWNTs under a transverse magnetic field are useful in the design of nano-drive device, nano-oscillator and actuators, nano-electron technology, and nano-composites absorbing wave, where carbon nanotubes act as basic elements.

\section{Dynamic coupled equations of MWNTs}

Effective investigations have been carried out on the mechanical properties of carbon nanotubes by using laminated shell models considering the van der Waals force between two walls (Yakobson et al 1996; Falvo et al 1997; Ru 2001;
Wang et al 2003). Motivated by these ideas, a set of concentric cylindrical shells with van der Waals interaction between adjacent walls can be used to model MWNTs under a transverse magnetic field, as shown in figure 1 . Here, a cylindrical coordinate system $(x, \theta, r)$ is introduced, in which $x$ expresses the longitudinal direction of laminated shells, $\theta$ the circumferential direction and $r$ the radial direction.

Applying a transverse magnetic field, $\tilde{B}_{0}\left(0,0, B_{0 r}\right)$ on the $j$ layer carbon nanotube with the cylindrical coordinate $(x$, $\theta, r)$, considering the axisymmetric displacement vector $\tilde{u}=$ $\left(u_{j}, 0, w_{j}\right)$ of the $i$ layer of carbon nanotube, and assuming that the magnetic permeability, $\eta$, of CNTs equals the magnetic permeability of the medium around it, the Lorentz force $\tilde{f}$ exerted on the CNTs, induced by the transverse magnetic field, can be written as (John 1984)

$$
f_{x}=\beta h_{j}\left(e_{\theta} B_{0 r}-\frac{\partial u_{j}}{\partial t} B_{0 r}^{2}\right) \quad f_{\theta}=0, \quad f_{r}=0 .
$$

In the above formula, $h_{j}$ is of MWNTs having single layer thickness, $\beta$ the conductivity of carbon nanotube, $e_{\theta}$ the circumferential electric field strength induced by perturbation of transverse magnetic field and $B_{0 r}$, exerted permeability on the carbon nanotube.

The corresponding electrodynamic equation of the $i$ th layer of MWNTs is given by (John 1984)

$$
\beta \eta\left(\frac{\partial e_{\theta}}{\partial t}-\frac{\partial^{2} u_{j}}{\partial t^{2}} B_{0 r}\right)-\frac{\partial^{2} e_{\theta}}{\partial x^{2}}=-\frac{2}{h_{j} \lambda} e_{\theta},
$$

where $\lambda$ represents the characteristic length of bending vibration of the MWNTs.

Based on axisymmetric shell model, the elastodynamic equation of the $i$ th layer of MWNTs is expressed as

$$
\begin{aligned}
& D_{N j}\left(\frac{\partial^{2} u_{j}}{\partial x^{2}}+\frac{\mu_{j}}{R_{j}} \frac{\partial w_{j}}{\partial x}\right)+\beta h_{j}\left(e_{\theta} B_{0 r}-\frac{\partial u_{j}}{\partial t} B_{0 r}^{2}\right) \\
& =\rho_{j} h_{j} \frac{\partial^{2} u_{j}}{\partial t^{2}} \\
& -D_{M j} \frac{\partial^{4} w_{j}}{\partial x^{4}}-\frac{D_{N j}}{R_{j}}\left(\frac{w_{j}}{R_{j}}+\mu_{j} \frac{\partial u_{j}}{\partial x}\right)+p_{j} \\
& =\rho_{j} h_{j} \frac{\partial^{2} w_{j}}{\partial t^{2}}
\end{aligned}
$$

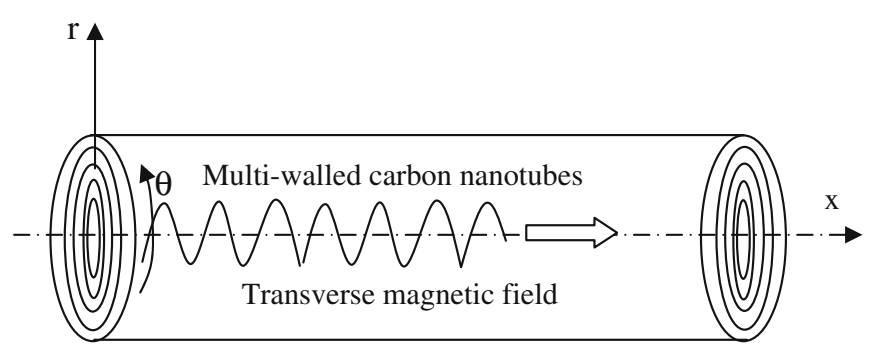

Figure 1. Multi-walled carbon nanotubes subjected to a transverse magnetic field. 
where $t$ is the time, $R_{j}$ the middle surface radius of the $j$ layer of multi walled carbon nanotube, $\rho_{j} h_{j}$ the mass density per unit lateral area of the $j$ layer, $p_{j}(j=1,2, \ldots . . N)$ the van der Waals pressure between two adjacent walls. In (3a) and (3b), $D_{N j}=\left(E_{j} h_{j}\right) /\left(1-\mu_{j}^{2}\right)$ and $D_{M j}=\left(E_{j} h_{j}^{3}\right) /$ $12\left(1-\mu_{j}^{2}\right)$ are the effective tensional and bending stiffness of the $j$ layer of MWNTs, where $E_{j} h_{j}$ the effective plane stiffness of the $j$ layer, $\mu_{j}$ the Poisson's ratio of the $i$ layer.

Utilizing (2) eliminates the circumferential electric field strength, $e_{\theta}$, in (3a), the dynamic coupled equation of the $i$ th layer of MWNTs under a transverse magnetic field is expressed as

$$
\begin{aligned}
& -\eta\left(D_{N j} \frac{\partial^{3} u_{j}}{\partial x^{2} \partial t}+D_{N j} \frac{\mu_{j}}{R_{j}} \frac{\partial^{2} w_{j}}{\partial x \partial t}-\rho_{j} h_{j} \frac{\partial^{3} u_{j}}{\partial t^{3}}\right) \\
& +\frac{1}{\beta}\left(D_{N j} \frac{\partial^{4} u_{j}}{\partial x^{4}}+D_{N j} \frac{\mu_{j}}{R_{j}} \frac{\partial^{3} w_{j}}{\partial x^{3}}-\rho_{j} h_{j} \frac{\partial^{4} u_{j}}{\partial x^{2} \partial t^{2}}\right) \\
& -\frac{2}{\beta h_{j} \lambda}\left(D_{N j} \frac{\partial^{2} u_{j}}{\partial x^{2}}+D_{N j} \frac{\mu_{j}}{R_{j}} \frac{\partial w_{j}}{\partial x}-\rho_{j} h_{j} \frac{\partial^{2} u_{j}}{\partial t^{2}}\right) \\
& +\left(\frac{2}{h_{j} \lambda} \frac{\partial u_{j}}{\partial t}-\frac{\partial^{3} u_{j}}{\partial t \partial x^{2}}\right) h_{j} B_{0 r}^{2}=0, \\
& -D_{M j} \frac{\partial^{4} w_{j}}{\partial x^{4}}-\frac{D_{N j}}{R_{j}}\left(\frac{w_{j}}{R_{j}}+\mu_{j} \frac{\partial u_{j}}{\partial x}\right)+p_{j} \\
& =\rho_{j} h_{j} \frac{\partial^{2} w_{j}}{\partial t^{2}} .
\end{aligned}
$$

The van der Waals interaction potential, as a function of the interlayer spacing between two adjacent walls, can be estimated by the Lennard-Jones model from the literature (Girifalco 1991). Since the innermost radius of carbon MWNTs is usually much larger than $0.5 \mathrm{~nm}$, the interlayer interaction potential between two adjacent walls can be simply approximated by the potential obtained for two flat graphite monolayers, denoted by $g(\delta)$, where $\delta$ is the interlayer spacing (Robertson et al 1992). This model has been previously shown to provide good agreement with experimental results carried out by $\mathrm{Lu}$ (1997). For the present infinitesimal buckling analysis, the van der Waals pressure, $p_{\mathrm{V}}(x, \theta)$, at any point between two adjucent tubes should be a linear function of the deflection jump at that point, so we have

$$
p_{\mathrm{V}}=\left[\frac{d g(\delta)}{d \delta}\right]_{\delta=\tau}+c(\Delta w),
$$

where

$$
c=\left.\frac{d^{2} g}{d \delta^{2}}\right|_{\delta=\tau} .
$$

In the above formula, $\tau$ is the initial interlayer spacing prior to buckling, which is equal or very close to the representative thickness of carbon SWNT, $(\Delta w)$ expresses the deflection jump due to buckling, and $c$ expresses the van der Waals interaction coefficient. Because the initial interlayer spacing is very close to the equilibrium interlayer spacing at which $d g(\delta) / d \delta=0$ described by Girifalco (1991), the first term of (5a) is negligible, reflecting the fact that all initial interlayer pressures vanish. Saito et al (2001) gave a van der Waals interaction coefficient as

$$
c=\frac{320 \mathrm{erg} / \mathrm{cm}^{2}}{0 \cdot 16 d^{2}}\left(d=1 \cdot 42 \times 10^{-8} \mathrm{~cm}\right) .
$$

Here, because the present analysis is limited to infinitesimal vibration, the interaction coefficient, $c$, is calculated at the initial interlayer spacing (about $0.34 \mathrm{~nm}$ ). The curvature-dependency of the interaction coefficient, $c$, is neglected here because it is very small when the innermost radii are much larger than $0.6 \mathrm{~nm}$ (Robertson et al 1992; Gulseren et al 2002). Thus, the van der Waals pressure $p_{j}(j=1,2, \ldots . . n)$ between two adjacent tubes can be expressed as

$$
\begin{aligned}
p_{1} & =p_{12}=c\left[w_{2}-w_{1}\right], \\
p_{2} & =p_{23}+p_{21}=c\left[w_{3}-w_{2}-\frac{R_{1}}{R_{2}}\left(w_{2}-w_{1}\right)\right], \\
p_{n-1} & =p_{(n-1) n}+p_{(n-1)(n-2)} \\
& =c\left[w_{n}-w_{n-1}-\frac{R_{n-2}}{R_{n-1}}\left(w_{n-1}-w_{n-2}\right)\right], \\
p_{n} & =p_{n(n-1)}=-c \frac{R_{n-1}}{R_{n}}\left[w_{n}-w_{n-1}\right] .
\end{aligned}
$$

Substituting van der Waals pressures $p_{j}(j=1,2, \ldots n)$ between two adjacent walls in (5) into (4a) and (4b), and considering that the tensional stiffness $D_{N j}=D_{N}$, the bending stiffness $D_{M j}=D_{M}$, the density $\rho_{j}=\rho$ and the thickness $h_{j}=h$ for every layer of MWNTs, the magnetic elastodynamic couple equations of MWNTs are expressed as

$$
\begin{aligned}
& -\eta\left(D_{N} \frac{\partial^{3} u_{1}}{\partial x^{2} \partial t}+\frac{D_{N} \mu}{R_{1}} \frac{\partial^{2} w_{1}}{\partial x \partial t}-\rho h \frac{\partial^{3} u_{1}}{\partial t^{3}}\right) \\
& +\frac{1}{\beta}\left(D_{N} \frac{\partial^{4} u_{1}}{\partial x^{4}}+\frac{D_{N} \mu}{R_{1}} \frac{\partial^{3} w_{1}}{\partial x^{3}}-\rho h \frac{\partial^{4} u_{1}}{\partial x^{2} \partial t^{2}}\right) \\
& -\frac{2}{\beta h \lambda}\left(D_{N} \frac{\partial^{2} u_{1}}{\partial x^{2}}+\frac{D_{N} \mu}{R_{1}} \frac{\partial w_{1}}{\partial x}-\rho h \frac{\partial^{2} u_{1}}{\partial t^{2}}\right) \\
& +h B_{0 r}^{2}\left(\frac{2}{h \lambda} \frac{\partial u_{1}}{\partial t}-\frac{\partial^{3} u_{1}}{\partial t \partial x^{2}}\right)=0,
\end{aligned}
$$




$$
\begin{aligned}
& D_{M} \frac{\partial^{4} w_{1}}{\partial x^{4}}+\frac{D_{N}}{R_{1}}\left(\frac{w_{1}}{R_{1}}+\mu \frac{\partial u_{1}}{\partial x}\right) \\
& +\rho h \frac{\partial^{2} w_{1}}{\partial t^{2}}+c w_{1}-c w_{2}=0, \quad j=1, \\
& -\eta\left(D_{N} \frac{\partial^{3} u_{j}}{\partial x^{2} \partial t}+\frac{D_{N} \mu}{R_{j}} \frac{\partial^{2} w_{j}}{\partial x \partial t}-\rho h \frac{\partial^{3} u_{j}}{\partial t^{3}}\right) \\
& +\frac{1}{\beta}\left(D_{N} \frac{\partial^{4} u_{j}}{\partial x^{4}}+\frac{D_{N} \mu}{R_{j}} \frac{\partial^{3} w_{j}}{\partial x^{3}}-\rho h \frac{\partial^{4} u_{j}}{\partial x^{2} \partial t^{2}}\right) \\
& -\frac{2}{\beta h \lambda}\left(D_{N} \frac{\partial^{2} u_{j}}{\partial x^{2}}+\frac{D_{N} \mu}{R_{j}} \frac{\partial w_{j}}{\partial x}-\rho h \frac{\partial^{2} u_{j}}{\partial t^{2}}\right) \\
& +h B_{0 r}^{2}\left(\frac{2}{h \lambda} \frac{\partial u_{j}}{\partial t}-\frac{\partial^{3} u_{j}}{\partial t \partial x^{2}}\right)=0 \\
& j=2,3, \cdots, n-1, \\
& D_{M} \frac{\partial^{4} w_{j}}{\partial x^{4}}+\frac{D_{N}}{R_{j}}\left(\frac{w_{j}}{R_{j}}+\mu \frac{\partial u_{j}}{\partial x}\right)+\rho h \frac{\partial^{2} w_{j}}{\partial t^{2}} \\
& -c w_{j+1}+c\left(1+\frac{R_{j-1}}{R_{j}}\right) w_{j}-c \frac{R_{j-1}}{R_{j}} w_{j-1}=0 \\
& j=2,3, \cdots, n-1, \\
& -\eta\left(D_{N} \frac{\partial^{3} u_{n}}{\partial x^{2} \partial t}+\frac{D_{N} \mu}{R_{n}} \frac{\partial^{2} w_{n}}{\partial x \partial t}-\rho h \frac{\partial^{3} u_{n}}{\partial t^{3}}\right) \\
& +\frac{1}{\beta}\left(D_{N} \frac{\partial^{4} u_{n}}{\partial x^{4}}+\frac{D_{N} \mu}{R_{N}} \frac{\partial^{3} w_{n}}{\partial x^{3}}-\rho h \frac{\partial^{4} u_{n}}{\partial x^{2} \partial t^{2}}\right) \\
& -\frac{2}{\beta h \lambda}\left(D_{N} \frac{\partial^{2} u_{n}}{\partial x^{2}}+\frac{D_{N} \mu}{R_{N}} \frac{\partial w_{n}}{\partial x}-\rho h \frac{\partial^{2} u_{n}}{\partial t^{2}}\right) \\
& +h B_{0 r}^{2}\left(\frac{2}{h \lambda} \frac{\partial u_{n}}{\partial t}-\frac{\partial^{3} u_{n}}{\partial t \partial x^{2}}\right)=0, \\
& D_{M} \frac{\partial^{4} w_{n}}{\partial x^{4}}+\frac{D_{N}}{R_{n}}\left(\frac{w_{n}}{R_{n}}+\mu \frac{\partial u_{n}}{\partial x}\right)+\rho h \frac{\partial^{2} w_{n}}{\partial t^{2}} \\
& +c \frac{R_{n-1}}{R_{n}} w_{n}-c \frac{R_{n-1}}{R_{n}} w_{n-1}=0, \quad j=n .
\end{aligned}
$$

Considering that the two ends of MWNTs under a transverse magnetic field are constrained by simple support, the couple vibration modes of the $j$ th layer of the MWNTs are given by

$$
\begin{array}{ll}
w_{j}(x, t)=A_{j} e^{s t} \sin \frac{m \pi}{l} x & (j=1,2, \cdots n), \\
u_{j}(x, t)=B_{j} e^{s t} \cos \frac{m \pi}{l} x & (j=1,2, \cdots n) .
\end{array}
$$

Substitution of (8a) and (8b) into (7) gives a set of algebraic equations for $A_{j}, B_{j}(j=1,2, \ldots . n)$, which is expressed as

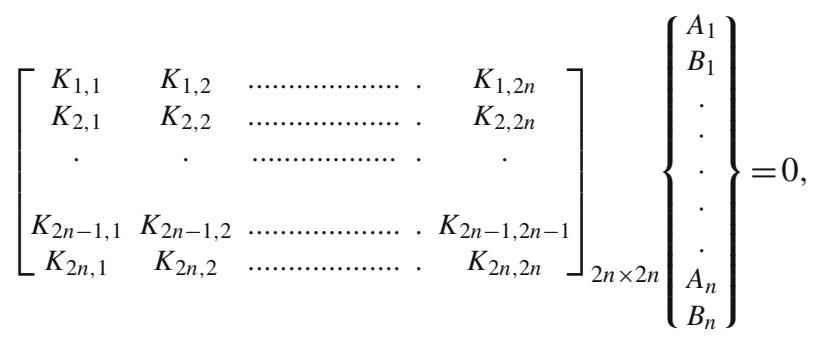

where the detailed expression of coefficients, $K_{j i}(j=1$, $2,3, \ldots 2 n ; i=1,2,3, \ldots 2 n$ ) in (9) is too lengthy and omitted in this paper. The frequency parameter, $s$, in (8a) and (8b) and the associated amplitude ratios $A_{1} / A_{2}$, $A_{1} / A_{3}, \ldots, A_{1} / A_{n}, B_{1} / B_{2}, B_{1} / B_{3}, \ldots, B_{1} / B_{n}$, for each frequency parameter, $s_{i}$, are determined by the condition for non-zero solution of $A_{1}, B_{1}, \ldots \ldots A_{n}, B_{n}$ in (9) as follows

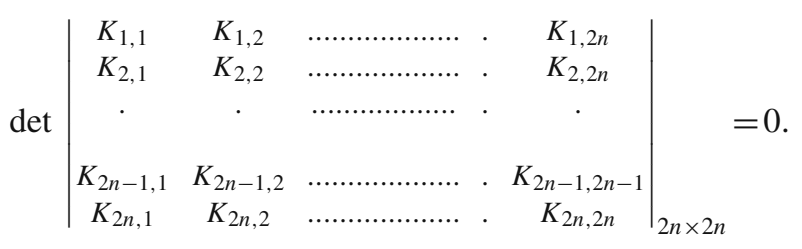

This condition determines the $n$ frequencies of $n$-walled CNTs subjected to a transverse magnetic field. Substituting each of the $n$ frequencies obtained into (9), we can determine the associated amplitude ratios $A_{1} / A_{2}, A_{1} /$ $A_{3}, \ldots, A_{1} / A_{n}, B_{1} / B_{2}, B_{1} / B_{3}, \ldots, B_{1} / B_{n}$. Thus, for an $n$-walled CNTs, there are totally $2 n$ vibration frequencies, each of which corresponds to a vibration mode.

\section{Example calculations and discussion}

As examples, dynamic characteristics of a double-walled CNTs and a four-walled CNTs, under a transverse magnetic field, are calculated. Here the strength of transverse magnetic field is given by $B_{0 r} /($ Tesla). For every single-wall of MWNTs, the thickness $h_{j}=h=0.34 \mathrm{~nm}\left(E_{j} h_{j}=E h=\right.$ $360 \mathrm{~J} / \mathrm{m}^{2}$ ), Poisson's ratio $\mu_{j}=\mu=0 \cdot 19$, the mass density per unit lateral area, $\rho_{j} h_{j}=\rho h=0.772 \times 10^{-7} \mathrm{~g} / \mathrm{cm}^{2}$, and $D_{M j}=D_{M}=0.85 \mathrm{eV}$ are taken. Here, a generalized vibration frequency of MWNTs under a transverse magnetic field is taken as (Falvo et al 1997; Wang et al 2005)

$$
f=\frac{\omega_{i}}{2 \pi C_{\mathrm{L}}} \quad\left(\mathrm{m}^{-1}\right)
$$

where $C_{\mathrm{L}}$ represents the speed of light. 
Firstly, a double-walled carbon nanotubes (DWNTs) with the innermost diameter, $R_{\min }=8.5 \mathrm{~nm}$, is used to calculate the effect of a transverse magnetic field on vibration characteristics of DWNTs. The two $n$-order frequency parameter, $\omega_{i}=\operatorname{Im}\left|s_{i}\right|$, of the DWNTs under a transverse magnetic field can be obtained by solving (10).

It is seen from figure 2 that the lowest frequency of DWNTs decreases as the strength of transverse magnetic field exerted on the DWNTs increases. Figure 3 shows that the strength of transverse magnetic field exerted on DWNTs does not change the highest frequency of DWNTs. From figure 4 , it is seen that the amplitude ratio $A_{1} / A_{2}$ of radial displacements and the amplitude ratio $B_{1} / B_{2}$ of axial displacements of DWNTs are constant when the strength of transverse magnetic field exerted on DWNTs is less than a

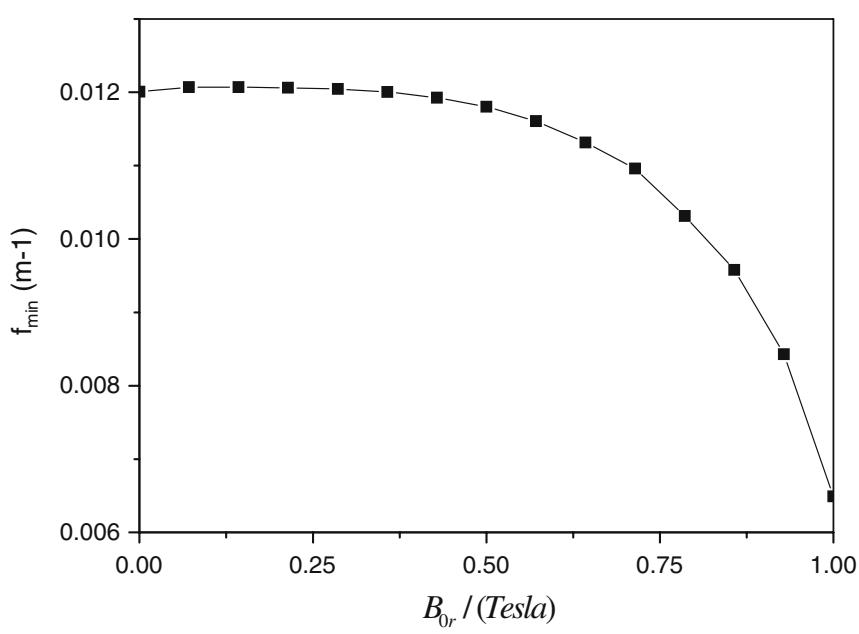

Figure 2. Effect of transverse magnetic fields on the lowest order frequency of DWNTs.

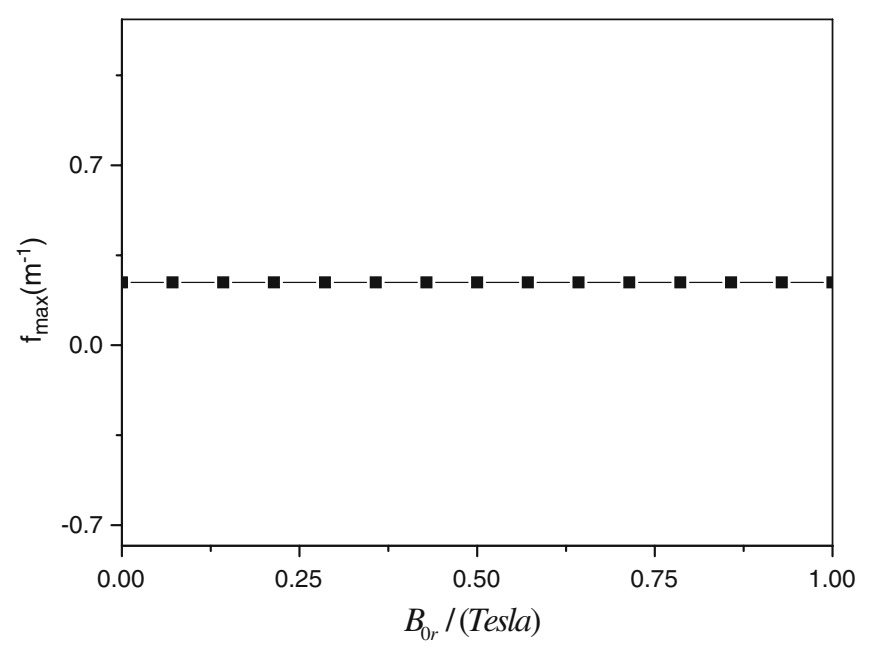

Figure 3. Effect of transverse magnetic fields on the highest order frequency of DWNTs. given value, where the axial amplitude ratio $A_{1} / A_{2}=1$ of DWNTs represents that the relative radial motion between two walls of the DWNTs does not appear, and the amplitude ratio $B_{1} / B_{2}$ of axial displacements is larger than 1 , which appears in an axially relative motion phenomena. When the strength of transverse magnetic field exerted on DWNTs is larger than 0.5 Tesla, the amplitude ratio $A_{1} / A_{2}$ of radial displacements and the amplitude ratio $B_{1} / B_{2}$ of axial displacements of DWNTs appear in relative motions and vehement oscillation.

Secondly, the dynamic characteristics of four-walled CNTs under transverse magnetic fields are described in figures $5-10$. It is seen from figure 5 that the lowest frequency of four-walled CNTs under transverse magnetic fields non-linearly decreases as the strength of transverse

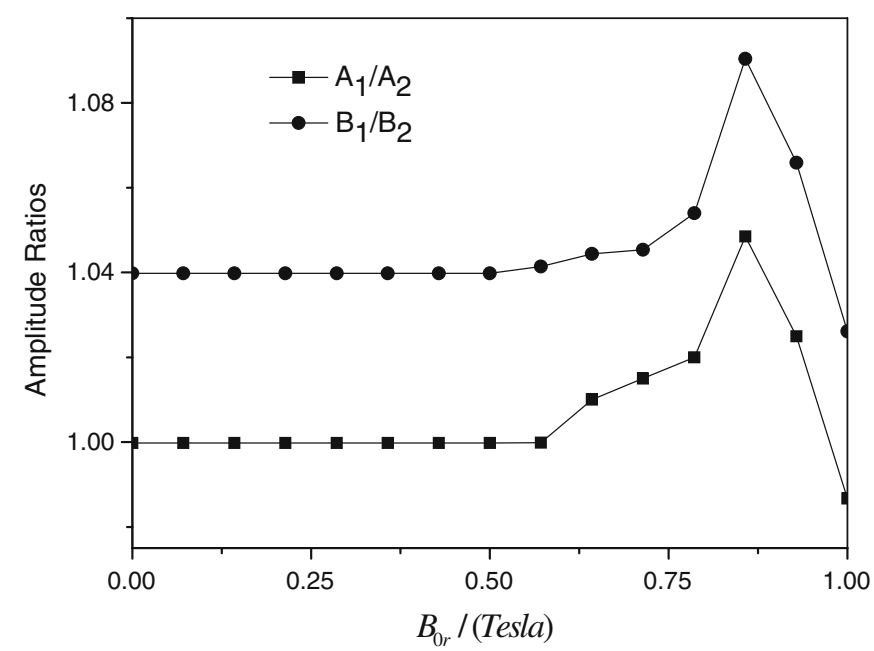

Figure 4. Amplitude ratios at the lowest frequency of DWNTs under transverse magnetic fields.

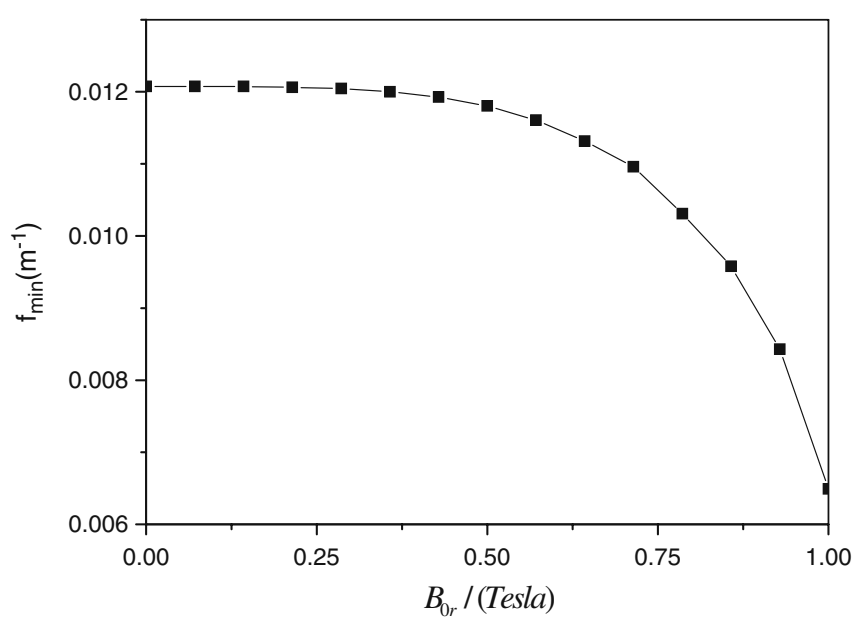

Figure 5. Effect of transverse magnetic fields on the lowest order frequency of four-walled CNTs. 
magnetic fields exerted on the four-walled CNTs increases. Through comparing figure 5 with figure 2 , it is seen that the effect of transverse magnetic fields on the lowest frequency of four-walled CNTs is less than that of DWNTs because the strength of van der Waals interaction in four-walled CNTs is larger than that in DWNTs. Figure 6 shows that the strength of transverse magnetic field exerted on four-walled CNTs does not affect the highest frequency of the four-walled CNTs, and the highest frequency of the four-walled CNTs is much higher than that of DWNTs.

Figures 7 and 8 describe, respectively the amplitude ratios, $A_{1} / A_{2}, A_{1} / A_{3}, A_{1} / A_{4}$, of radial displacements and

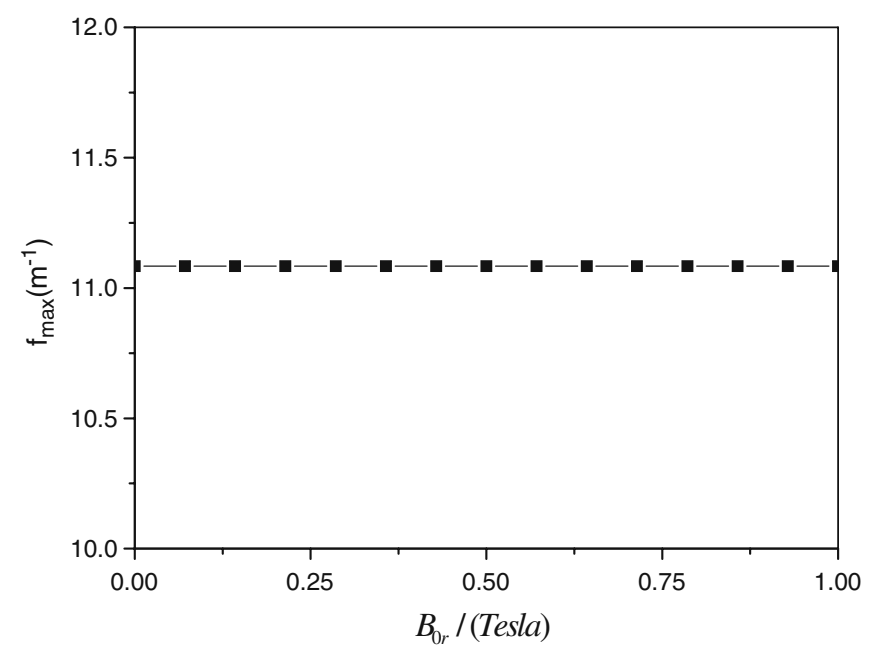

Figure 6. Effect of transverse magnetic fields on the highest frequency of four-walled CNTs.

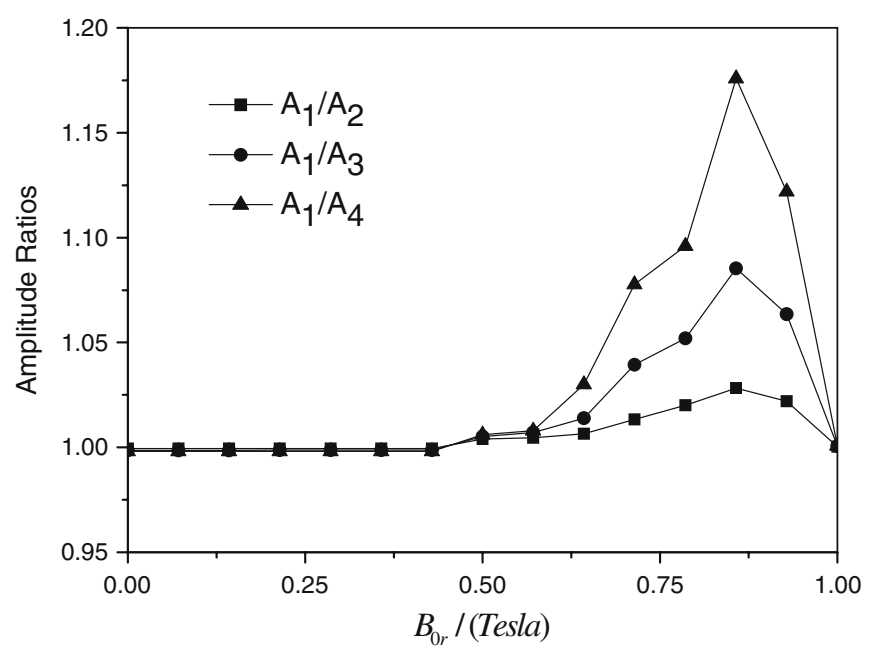

Figure 7. Amplitude ratios of radial displacement at the lowest frequency of four-walled CNTs under transverse magnetic fields. the amplitude ratios $B_{1} / B_{2}, B_{1} / B_{3}, B_{1} / B_{4}$ of axial displacements of four-walled CNTs. It is seen from figure 7 that two walls of the four-walled CNTs appear in larger relative radial motion and axial motion when the strength of transverse magnetic field exerted on the four-walled CNTs is larger than 0.375 Tesla, and the radial amplitude ratio between two walls of the four-walled CNTs is less than its axial amplitude ratio.

Figures 9 and 10 show, respectively that effects of the length radial ratio of MWNTs on the amplitude ratios of radial displacement and axial displacement at the lowest frequency of four-walled CNTs subjected to the strength of transverse magnetic field, $B_{0 r} /($ Tesla $)=0 \cdot 5$. It is seen from

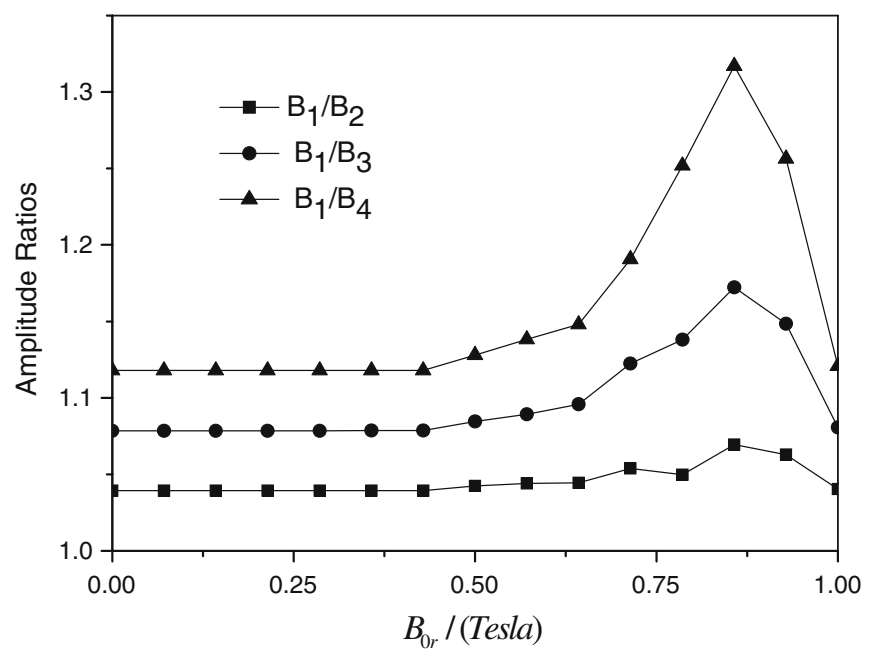

Figure 8. Amplitude ratios of axial displacement at the lowest frequency of four-walled CNTs under transverse magnetic fields.

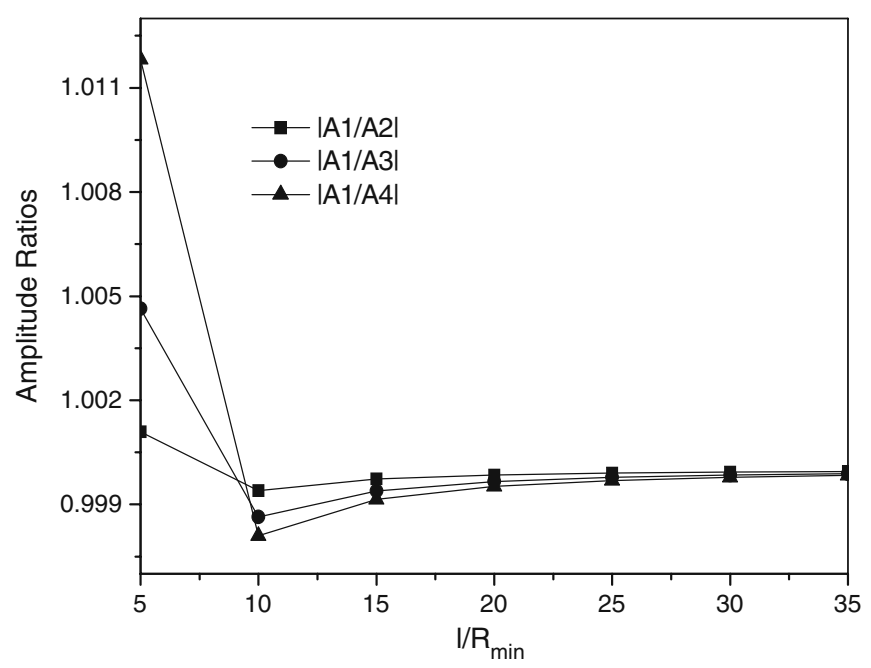

Figure 9. For different lengths of radial ratios, the amplitude ratios of radial displacement at the lowest frequency of four-walled CNTs subjected to a transverse magnetic field. 


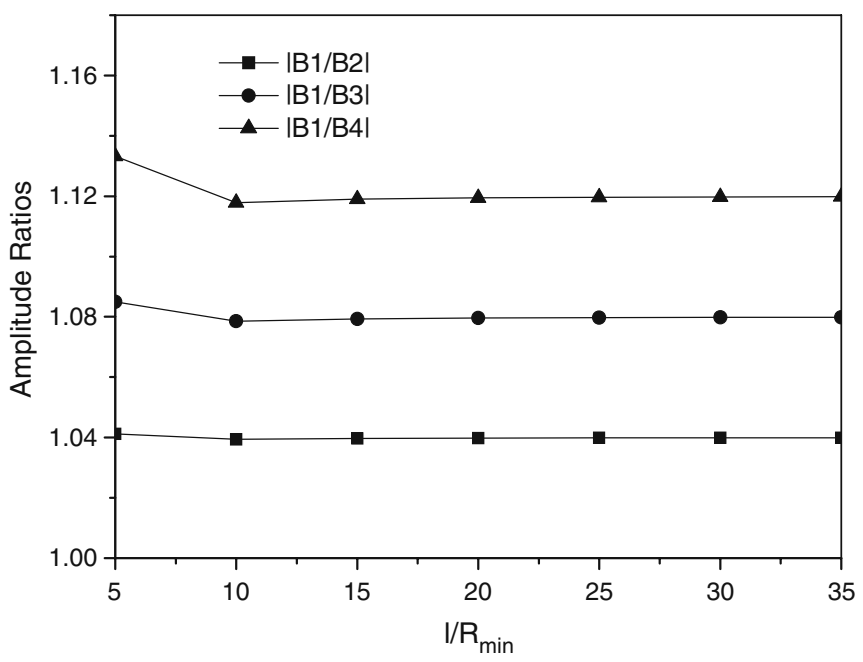

Figure 10. For different lengths of radial ratios, the amplitude ratios of axial displacement at the lowest frequency of four-walled CNTs subjected to a transverse magnetic field.

figures 9 and 10 that the amplitude ratios $A_{1} / A_{2}, A_{1} / A_{3}$, $A_{1} / A_{4}$ between two walls of radial displacements and the amplitude ratio $B_{1} / B_{2}, B_{1} / B_{3}, B_{1} / B_{4}$, of axial displacements of four-walled CNTs appear in relative motions and vehement oscillation when the length radial ratio of MWNTs is less than 10 . The smaller the length radial ratio of four-walled CNTs, the larger the relative radial motion and axial motion between two walls of the four-walled CNTs.

Because a few detailed reports on vibration frequencies and modes of CNTs under the coupled magnetic field and electric field are available in literature so far, an exact comparison of the present result with existing experimental results is difficult, and should be done in the future. Here, a simple validation of the present method is given by comparing between the free vibration frequency of simple supported MWNTs with the larger ratio of length to radius using the present method and the low frequency Raman modes of a MWNT obtained by the atomistic model (Benoit et al 2002). When the computing parameters for two models are taken as identical, the vibration frequency for the low Raman modes of five-wall CNTs with small innermost diameter $d_{1}=1.2 \mathrm{~nm}$ is about $199\left(\mathrm{~cm}^{-1}\right)$ (Benoit et al 2002), and the free vibration frequency of a simple supported MWNTs with the larger ratio of length to radius using the present method is $208 \mathrm{~cm}^{-1}$, in which the excellent agreement between two results is seen.

\section{Conclusions}

This paper presents an analytical method to study the effects of transverse magnetic fields on vibration characteristics of MWNTs for which detailed experimental data or molecular dynamic simulations are not yet available in the literature. Main conclusions are given by

(I) The transverse magnetic field exerted on MWNTs makes the lowest frequency of the MWNTs non-linearly decrease and the highest frequency of the MWNTs being unaffected.

(II) Two walls of MWNTs appear in larger relative radial motion and axial motion when the strength of transverse magnetic fields exterted on the MWNTs is larger than a given value, and the relative radial motion amplitude between two walls of the MWNTs is less than its relative axial motion amplitude.

(III) The smaller the length radial ratio of MWNTs, the larger the relative radial motion and axial motion between two walls of the MWNTs.

(IV) Because electronic and transport properties of carbon nanotubes could be extremely sensitive to the dynamic frequencies and relative motion between two walls of carbon nanotubes under transverse magnetic field, the new features, and interesting numerical results of the present work may stimulate further interest in this topic.

\section{Acknowledgements}

The authors thank the support of the National Science Foundation of China under Number 10872127 and 10932007, and are grateful to reviewers for valuable suggestions and comments.

\section{References}

Avouris P H, Hertel T, Martel R, Schmidt T, Shea H R and Walkup R E 1999 Appl. Surf. Sci. 141201

Benoit J M, Buisson J P, Chayvet O, Godon C and Lefrant S 2002 Phys. Rev. B66 073417

Cai H and Wang X 2006 Nanotechnology 1745

Dresselhaus M S, Dresselhaus G and Avouris P H 2001 Top. Appl. Phys. 80287

Falvo M R, Clary G J, Taylor R M, Chi V, Brooks F P, Washburn S and Superfine R 1997 Nature 389582

Fedorov G, Barbara P, Smirnov D, Jiménez D and Roche S 2010 Appl. Phys. Lett. 96132101

Girifalco L A 1991 J. Phys. Chem. 955370

Gulseren O, Yildirim T and Ciraci S 2002 Phys. Rev. B65 153405

Han Q, Lu G and Dai L 2005 Compos. Sci. Technol. 651337

Heremans J, Olk C H and Morelli D T 1994 Phys. Rev. B49 15122

Iijima S 1991 Nature 35456

John K D 1984 Electromagnetics (New York: McGraw Hill, Inc.)

Kandaa Akinobu, Uryub Seiji, Tsukagoshib Kazuhito, Ootukaa Youiti and Aoyagib Yoshinobu 2002 Physica B323 246

Kibis O V 2002 Physica E: Low-Dimensional Systems and Nanostructures 12741

Langer L, Bayot V, Grivei E and Issi J -P 1996 Phys. Rev. Lett. 76 479

Lau K T 2003 Chem. Phys. Lett. 370399 
Li X and Bhushan B 2002 Mater. Charact. 4811

Li X, Chang W C, Chao Y J, Wang R and Chang M 2004 Nano Letts. 4613

Lu J 1997 Phys. Rev. Lett. 791297

Macedo C A and Souza A M C 2004 Physica B354 290

Nardelli M B, Brabec C and Maiti A 1998 Phys. Rev. Lett. 80 313

Popov V N and Henrard J P 2002 Phys. Rev. B65 235415

Qin C, Shen J F, Hu Y Z and Ye M X 2009 Compos. Sci. Technol. 69427

Reddy A, Leela Mohanal, Shaijumon M M and Ramaprabhu S 2006 Nanotechnology 175299

Robertson D H, Brenner D W and Mintmore J W 1992 Phys. Rev. B45 12592

Ru C Q 2000 Phys. Rev. B62 9973

Ru C Q 2001 J. Mech. Phys. Solids 491265

Saito R, Takeya T and Kimura T 1998 Phys. Rev. B57 4145
Saito R, Matsuo R, Kimura T, Dresselhaus G and Dresselhaus M S 2001 Chem. Phys. Lett. 348187

Steven G L 2001 Top. Appl. Phys. 80113

Tu Z C and Yang Z C 2002 Phys. Rev. B65 233

Wang C Y, Ru C Q and Mioduchowski A 2003 Int. J. Solids Struct. 403893

Wang C Y, Ru C Q and Mioduchowski A 2004 J. Appl. Mech. 71 622

Wang C Y, Ru C Q and Mioduchowski A 2005 J. Appl. Phys. 97 024310

Wang X and Cai H 2006 Acta Mater. 542067

Wang Y and Ni X 2003 Chin. Phys. 121007

Yakobson B I, Brabec C J and Bernholc J 1996 Phys. Rev. Lett. 76 2511

Yakobson B I and Avouris Phaedon H 2001 Top. Appl. Phys. 80287

Zhang L, Zhu H, Song Y, Zhang Y M and Huang Y 2008 Mater. Sci. Eng. B: Solid 15378 